Proc. of 12th International Workshop on Positron and Positronium Chemistry, August 28-September 1, 2017, Lublin, Poland

\title{
Track Effects in Positronium Formation
}

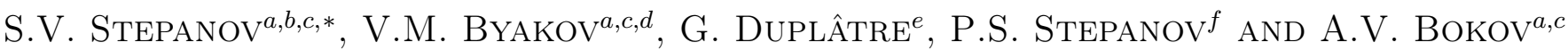 \\ ${ }^{a} \mathrm{NRC}$ "Kurchatov Institute" - Institute of Theoretical and Experimental Physics, \\ B. Cheremuskinskaya str., 25, 117218 Moscow, Russia \\ ${ }^{b}$ National Research Nuclear University "MEPhI", 115409, Moscow, Russia \\ ${ }^{c}$ Lomonosov Moscow State University, Chemical Department, Leninskie Gory, Moscow, Russia \\ ${ }^{d}$ D. Mendeleyev University of Chemical Technology of Russia, Miusskaya sq., 9, 125047, Moscow, Russia \\ ${ }^{e}$ Institut Pluridisciplinaire Hubert Curien, CNRS/IN2P3, BP 2867037 Strasbourg, France \\ ${ }^{f}$ Department of Physics and Astronomy, 104 Overmann Hall, Bowling Green State University, OH, 43403, USA
}

\begin{abstract}
We discuss some aspects important for interpretation of the Ps formation process in liquids and molecular media: (1) inhomogeneity of intratrack reactions and parameters of the $\mathrm{e}^{+}$track, (2) final states of $\mathrm{e}^{+}$, its solvation in polar and nonpolar liquids, relation to $\mathrm{e}^{+}$mobility, (3) quasi-neutrality of the $\mathrm{e}^{+}$blob and its ambipolar outdiffusion, (4) appearance of the "in-blob" and "out-of-the-blob" positron fractions, and (5) electric field effect on Ps formation.
\end{abstract}

DOI: 10.12693/APhysPolA.132.1461

PACS/topics: positron, positronium, annihilation, ion-electron recombination, solvation, mobility, external electric field, track effects, polar and nonpolar liquids

\section{Introduction}

All early radiolytic processes are inhomogeneous. Ionization of molecules is the main channel of the energy losses of any fast (but not ultrarelativistic) charged particle moving in matter. The slowing-down particle forms a track along its trajectory. Such a track is a chain of local clusters of chemically active primary radiolysis products - ion-electron pairs, excited states etc. [1]. Taking into account this spatial inhomogeneity is important for a correct description of the intratrack processes.

Ionization slowing-down ceases when the velocity of the particle becomes less than that of the external (valence) electrons of the molecules of the medium. Projectile electrons $\left(\mathrm{e}^{-*}\right)$ stop to ionize when their energy $E\left(\mathrm{e}^{-*}\right)$ becomes less than the first ionization potential $I$ of the surrounding molecules, i.e. when $E\left(\mathrm{e}^{-}\right) \leq 10 \mathrm{eV}$. Muons and protons become subionizing at the same velocity as the electrons. However their energy significantly exceeds the ionization potential in the ratio of the mass of the particle, $m$, to that of the electron, $m_{\mathrm{e}}$. Ionization threshold energies for muon and proton are respectively equal to: $E(\mu)=2 \mathrm{keV}, E(\mathrm{p})=20 \mathrm{keV}, m_{\mu} / m_{\mathrm{e}} \approx 200$, $m_{\mathrm{p}} / m_{\mathrm{e}} \approx 2000$.

Generally, the Ps formation process depends on many parameters such as electron and positron affinities towards the solvent/solute molecules, their polarity (presence of a dipole moment), $\mathrm{e}^{+}$and $\mathrm{e}^{-}$work functions and their mobilities, "strength" of the quasifree positronium (its binding energy) etc. [2,3]. Therefore, sometimes it is fairly difficult to figure out common features of this pro-

\footnotetext{
*corresponding author; e-mail: stepanov@itep.ru
}

cess even in neat media. Here we shall consider only some aspects, for which there is clear experimental evidence.

\section{Parameters of the $\mathrm{e}^{+}$track}

In Fig. 1 there are shown the slowing-down times and ranges of fast electrons in liquid water. Similar values are expected for the positrons.
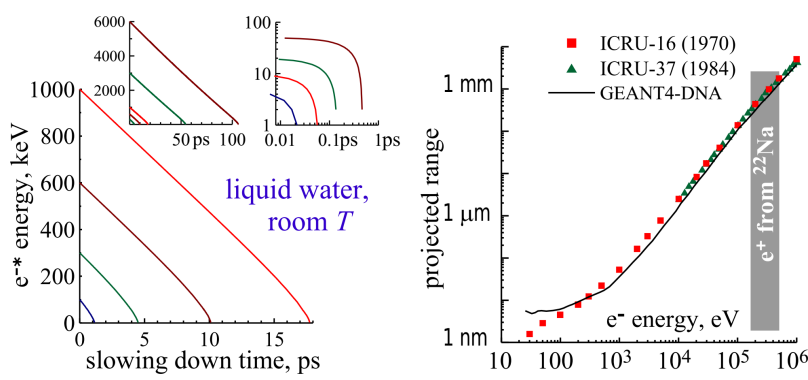

Fig. 1. Typical parameters of fast electrons in water [ESTAR: Stopping Powers and Ranges for Electrons]. Solid line (GEANT4-DNA) on the right plot is taken from [4].

To discuss the formation of the Ps atom, the parameters of the final section of the positron track are most important, when the rate of its ionization energy loss is maximal $(\sim \mathrm{eV} / \AA)$. This is the so-called $\mathrm{e}^{+}$blob. In this region is the Bragg peak of the positron. Theoretical estimates and modeling in the GEANT4 toolkit show that the positron blob is a fairly compact spheroidal region containing several tens of ion-electron pairs whose spatial distribution can be described by a Gaussian, $\exp \left(-r^{2} / a^{2}\right)[5]$. In water, where $a \approx 40 \AA$, the rootmean-square diameter of the blob, $2\left(\left\langle r^{2}\right\rangle^{1 / 2}\right) \approx 2.44 a \approx$ $100 \AA[6]$. 
By the end of ionization slowing down most intrablob electrons are produced with an initial kinetic energy about the ionization potential $I \approx 10 \mathrm{eV}$. These subionizing electrons lose their energy by exciting molecular vibrations (thermalization process).

\section{Solvation and mobility}

In polar media the duration of thermalization is limited by the solvation process. Electrons and positrons trapped in shallow traps acquire a solvation shell, orienting around themselves the dipole moments of the nearest molecules [1, 2]. In many liquids (for example, alcohols), the duration of electron solvation takes tens of picoseconds. In water, the hydration time is only $0.3 \mathrm{ps.} \mathrm{As}$ a result, the mobility of an electron falls by several orders of magnitude, actually the diffusion coefficient of the quasifree electron is $D_{q f} \sim \lambda v / 6 \sim \hbar / m \sim 1 \mathrm{~cm}^{2} / \mathrm{s}$ (here $\lambda=2 \pi \hbar / m v$ is the de Broglie wave length, $v$ is the thermal velocity; if $D_{q f}=\mu T / e=1 \mathrm{~cm}^{2} / \mathrm{s}$; mobility is $\mu=40 \mathrm{~cm}^{2} /(\mathrm{V} \mathrm{s}), e$ is the electric charge of the $\mathrm{e}^{+}$) and that of the solvated particle is $D_{s} \sim T / 4 \pi \eta R \sim$ $10^{-5} \mathrm{~cm}^{2} / \mathrm{s}$, where $\eta$ is the viscosity and $R$ is the radius of the solvated $\mathrm{e}^{-}$.

It is usually considered that in nonpolar media solvation is rather weak. In normal hydrocarbons the solvation is due to a peculiar orientation of molecules (which have a rod-like shape) with respect to the direction of the excess particle electric field $\left(\mathrm{e}^{-}, \mathrm{e}^{+}\right.$, ions). It is related with the anisotropic polarizability of such rod-like molecules. Although the solvation times in polar and nonpolar liquids are comparable, a respective energy gain in normal hydrocarbons is much less than in polar media. So the mobility of the solvated $\mathrm{e}^{-}$or $\mathrm{e}^{+}$is orders of magnitude higher than in polar liquids. In nonpolar liquids with spherically-symmetric molecules (like neopentane, tetramethylsilane etc.) even this solvation effect (related with the anisotropy of polarization) is absent. Therefore, it is believed that in these liquids electrons and positrons are in a quasi-free state. Their mobilities are much greater than in normal hydrocarbons. Positrons (as well as excess electrons) exist there in the quasi-free state during all their life up to annihilation. As an exception, one may point out $\mathrm{C}_{6} \mathrm{~F}_{6}$, where positron forms a slightly bound state with fluorine atoms.

In nonpolar media the $\mathrm{e}^{+}$mobility can be determined directly from the measurement of its drift velocity in an external electric field. The drift velocity is directly proportional to the shift of the peak of the $511 \mathrm{keV}$ annihilation photons, Fig. 2.

A priori, the existence of a positron in a highly mobile state during a long time should result in an extended duration of the Ps formation process (so-called delayed Ps formation). In fact, this does not happen, because the positron escapes its blob very quickly, leaving behind the intrablob electrons. The latter are localized in the blob because of the Coulomb attraction to the positively charged parent ions. The positron that left the blob, may

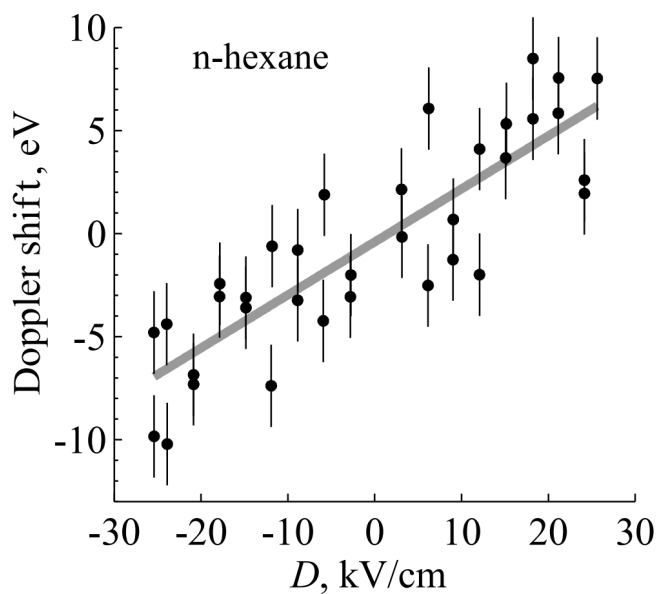

Fig. 2. Dependence of the Doppler shift of the peak of the annihilation photons in $n$-hexane vs. applied electric field [7].

increase the Ps formation probability only if preexisting trapped electrons are disseminated in the medium. Such electrons can be formed, for example, during preceding irradiation of the medium (gammas from the $\mathrm{e}^{+}$source) and their trapping in shallow structural traps [8].

\section{Why is $\mathrm{e}^{+}$mobility much larger than the mobility of excess electrons?}

In nonpolar media, when the de Broglie wavelength of the particles exceeds molecular dimensions, the mobility of $\mathrm{e}^{+}$and $\mathrm{e}^{-}$is determined by their scattering on local fluctuations of the bottom of the conduction band. In fact, these fluctuations are directly related to fluctuations of the density of the medium. Apparently, the reason is that the dominant interactions of $\mathrm{e}^{+}$and $\mathrm{e}^{-}$with the medium have different signs: ground state energies for $\mathrm{e}^{-}$are mostly negative, but positive for $\mathrm{e}^{+}$, because the electrons are attracted to atomic nuclei whereas positrons are repelled from them. Therefore, negative density fluctuations ("voids") look like potential barriers for excess electrons, but for positrons they are potential wells (it is easier to pass through them). For the same reason, preferential traps for $\mathrm{e}^{+}$are voids (vacancies in solids), in contrast to electrons, Table I. Anyway, this question requires further investigations.

TABLE I

Mobilities of $\mathrm{e}^{+}$and $\mathrm{e}^{-}$in some non-polar media [7, 9].

\begin{tabular}{c|c|c}
\hline \hline Medium & $\mu^{+}\left[\mathrm{cm}^{2} /(\mathrm{V} \mathrm{s})\right]$ & $\mu^{-}\left[\mathrm{cm}^{2} /(\mathrm{V} \mathrm{s})\right]$ \\
\hline isooctane & $134(6)$ & $4.5-7$ \\
n-hexane & $100(9)$ & $0.07-0.09$ \\
c-hexane & 100 & 0.4 \\
hexafluorobezene & $0(7)$ & 0.01 \\
benzene & $19.5(6)$ & $0.11-0.14$ \\
polyethylene & $43 ; 58$ & $\sim 0.1$
\end{tabular}




\section{Simplest consideration of the effect of the external electric field on Ps formation}

Confirmation that the $\mathrm{e}^{+}$blob is an essentially multiparticle object comes also from experiments on the effect of an external electric field $D$ on the total positronium formation probability (Ps yield) $P_{\mathrm{Ps}} \approx I_{3}+I_{1} \approx 4 I_{3} / 3$ in both its ortho- and para-spin states. In Fig. 3 there are shown several typical field dependences of the Ps yield.
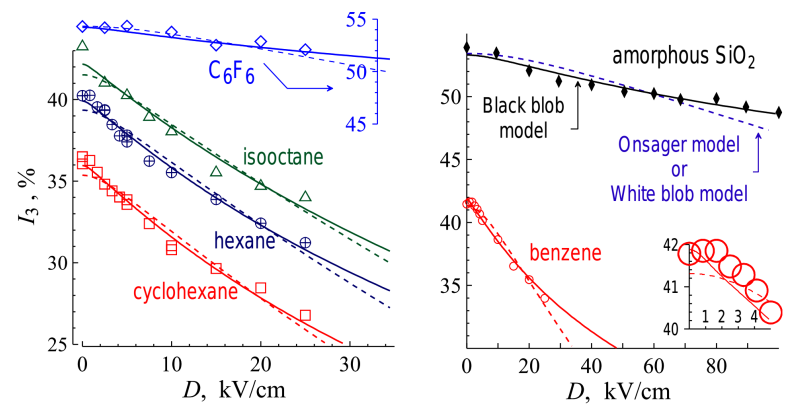

Fig. 3. Dependence of the ortho-Ps formation probability vs. the external electric field $D$ in some nonpolar media at room temperature $[10,11]$. Dashed lines show fits according to the "white" blob model, Eq. (2) Solid lines represent the fit according to the "black" blob model, which accounts for the "in-blob" and "out-ofblob" positron fractions (see Sect. 8).

The traditional approach to interpret the effect of an external electric field $D$ on the Ps yield is based on application of the Onsager Eq. (1) for the probability $P_{g r}$ of a geminate recombination of ions (or an ion-electron pair), to the electron-positron recombination. Such an approach assumes that Ps is formed not in a blob with many ion-electron pairs, but in a "spur" containing a positron $\mathrm{e}^{+}$and one electron (presence of a positive ion is neglected in fact) [12], that is $P_{\mathrm{Ps}} \approx P_{g r}$ :

$$
P_{g r}=1-\left(1+\frac{e D r_{c}}{2 T}\right) \exp \left(-\frac{r_{c}}{r_{0}}\right), \quad r_{c}=\frac{e^{2}}{\varepsilon T} .
$$

Here $r_{0}$ is the "initial" distance between thermalized $\mathrm{e}^{+}$ and $\mathrm{e}^{-}$in the terminal spur, and $r_{c}$ is the Onsager radius, that is, the distance at which the Coulomb energy of attraction between $\mathrm{e}^{+}$and $\mathrm{e}^{-}$is equal to the thermal energy, $k_{\mathrm{B}} T$. For nonpolar media with the dielectric permittivity $\varepsilon \approx 2$ at room temperature $r_{c} \approx 300 \AA$. From Fig. 3 it can be seen that in the absence of a field $(D=0)$ the Ps yield (para- and ortho-spin states) is approximately 0.5 :

$$
P_{\mathrm{Ps}}(D=0) \approx P_{g r}(D=0)=1-\exp \left(-\frac{r_{c}}{r_{0}}\right) \approx 0.5 .
$$

So we obtain that $r_{0} \approx 1.4 r_{c} \approx 400 \AA$.

On the other hand, if now we try to describe the experimentally observed slope of the positronium formation probability vs. applied electric field $D$, then we have to adopt that the Onsager radius is $7-8$ times less! Here we are facing a huge contradiction. It is eliminated by using the $\mathrm{e}^{+}$blob model [11], which in the case of small $D$ gives for $P_{\mathrm{Ps}}$ a very similar expression, but with a different physical meaning of the parameters involved

$$
\begin{aligned}
& P_{\mathrm{Ps}}=1-\left(1+\frac{e D \tilde{r}_{c}}{2 \varepsilon T}\right) \exp \left(-\frac{\tilde{r}_{c}}{\tilde{r}_{0}}\right), \\
& \tilde{r}_{c} \approx 80=n_{0} R_{e p} \Rightarrow \tilde{r}_{0} \approx 1.4 \tilde{r}_{c} \approx 100 \approx a_{p} .
\end{aligned}
$$

Here $\tilde{r}_{c}$ is the product of the initial number of ionelectron pairs in the blob, $n_{0} \approx 30-40[3,6]$, by the reaction radius of the Ps formation, $R_{e p}$. A rough comparison with experiment, Fig. 3, leads to $R_{e p} \approx 2 \AA$ and $\tilde{r}_{0} \approx 100 \approx a_{p}\left(\tilde{r}_{0}\right.$ has a meaning of $a_{p}$, which characterizes the $\mathrm{e}^{+}$spatial distribution just after the ionization slowing down, $\left.\sim \exp \left(-r^{2} / a_{p}^{2}\right)\right)$. Such a small value of $R_{e p}$ indicates that this process of the quasifree Ps formation is not strictly diffusion-controlled. It is also accompanied by some energy release from the $\mathrm{e}^{+} \mathrm{e}$ pair to the medium, which hampers Ps formation.

Thus, we come to the conclusion that in order to explain the influence of the external field on $P_{\mathrm{Ps}}$, it is necessary to consider that the blob is an essentially multiparticle object, which should be described by equations of inhomogeneous chemical kinetics.

\section{Spherical symmetry of the terminal positron blob}

On the basis of the results shown in Fig. 4 (that Ps formation decreases in the same way irrespectively on the direction of the applied electric field with respect to the $\mathrm{e}^{+}$implantation direction) in $[13,14]$ it was concluded than the structure of the terminal positron blob is spherically symmetric, by contrast with the asymmetric structure at the end of the muon track [15].
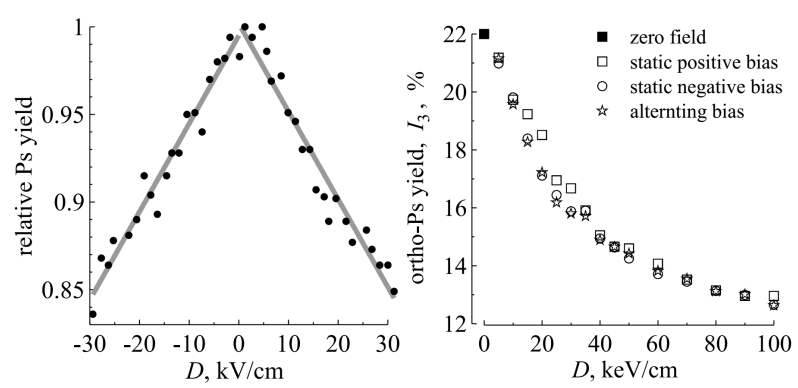

Fig. 4. Left: symmetric decrease of the Ps yield for positive and negative direction of the electric field towards positron implantation [13]. Right: decrease of the ortho-Ps formation vs. external electric field in lowdensity polyethylene [14].

\section{Absence of $E$-field effect on $P_{\mathrm{Ps}}$ in polar media}

Firstly, the electric field is greatly attenuated (78 times, in water) when it penetrates into the polar media. Secondly, after the positron gets solvated, its mobility 
falls by several orders of magnitude. Such $\mathrm{e}^{+}$cannot be moved by the field over a significant distance. For example, if the field inside the medium is $10 \mathrm{kV} / \mathrm{cm}$, and the $\mathrm{e}^{+}$mobility is $0.01 \mathrm{~cm}^{2} /(\mathrm{V} \mathrm{s})$ (overestimated), then, during $1 \mathrm{~ns}$, the positron is displaced only by $10 \AA$ (really less). These circumstances explain the inefficiency of an electric field in polar liquids.

\section{Quasi-neutrality and ambipolar out-diffusion}

Just after the last ionization event, all intrablob species (including $\mathrm{e}^{+}$) are arranged within the terminal blob. Their spatial distribution can be described by a Gaussian function $\exp \left(-r^{2} / a^{2}\right), a \approx 40 \AA$. However, the blob species are still "hot" (the particles inside are not thermalized). They lose energy by exciting molecular vibrations. Intrablob electrons are trying to escape out of the blob, but attraction to the immobile positive ions prevents their out-diffusion. Track electrons have to screen them and adjust their motion to the distribution of ions (quasi-neutrality condition). This results in the appearance of a local electric field between ions and track electrons (similarly to a spherical capacitor). That is why further diffusion expansion of the terminal blob proceeds rather slowly. It obeys the law of ambipolar diffusion, when the diffusion coefficient of this expansion is equal to the double diffusion coefficient of the positive ions $\left(10^{-5}-10^{-4} \mathrm{~cm}^{2} / \mathrm{s}\right)[11]$. This results in the appearance of the local intrablob electric field $\boldsymbol{E}$ which is directed outside the blob. The out-diffusion flux of electrons is counter-balanced by their drift flux in this local field: $-D_{\mathrm{e}} \nabla c_{\mathrm{e}}-\mu_{\mathrm{e}} \boldsymbol{E} c_{\mathrm{e}}=0$.

\section{Localization of the thermalized positrons: in-blob and out-of-the-blob fractions}

The intrablob field partially promotes the escape of $\mathrm{e}^{+}$ out of the blob, which results in the formation of the $\mathrm{e}_{\text {out }}^{+}$fraction, Fig. 5. Those positrons which stop within the blob $\left(\mathrm{e}_{i n}^{+}\right)$become trapped inside it due to the Debye screening of the $\mathrm{e}^{+}$by other charged species. This results in a positron binding energy $\approx 0.1-0.3 \mathrm{eV}$ towards its blob [9]. Because this value is rather large (in comparison with the temperature, $k_{B} T$ ), the in-blob positrons cannot escape out of the blob and are not affected by an external electric field, so $\mathrm{e}_{\text {out }}^{+}$is the only field dependent fraction. The "in-blob" positrons mainly form Ps, because they easily meet intrablob electrons and react with them: $\mathrm{e}_{\text {in }}^{+}+\mathrm{e}^{-} \Rightarrow P s$. On the contrary, $\mathrm{e}_{\text {out }}^{+}$mostly annihilate as "free $\mathrm{e}^{+}$" into 2-gammas. The model which takes into account resistance of these two positron fractions is called the black blob model [9]. As follows from Fig. 3 it agrees better with the experimental on Ps formation probabilities measured at different $E$-fields.

Note that applying an external field (for example, $30 \mathrm{kV} / \mathrm{cm}$ ) is not enough to extract $\mathrm{e}_{i n}^{+}$from the blob, since the work of this field over the positron is $30 \mathrm{kV} / \mathrm{cm} \times 100 \approx 0.03 \mathrm{eV}$, which is much less than
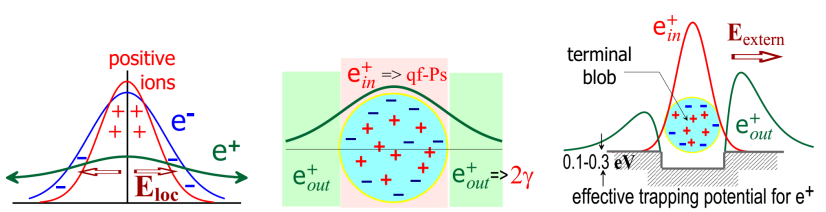

Fig. 5. Appearance of the intrablob electric field and formation of in-blob and out-of-the-blob positron fractions.

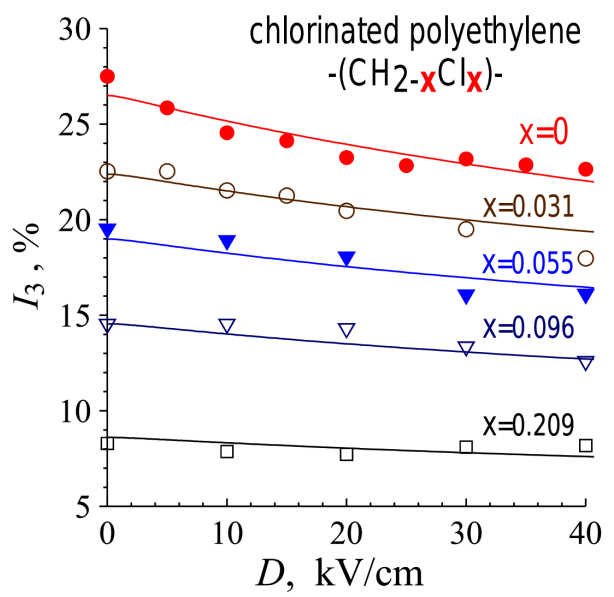

Fig. 6. Decrease of the ortho-Ps yield vs. $D$ in chlorinated polyethylene [9]. Solid line - fit on the basis of the black blob model.

the $\mathrm{e}_{i n}^{+}$binding energy. Therefore, an external field can only affect on those out-of-blob positrons, which due to the diffusion flux (directed inside the blob) may return in it, meet electrons there and form Ps.

The existence of two $\mathrm{e}^{+}$fractions manifests itself in the Ps formation process in many media, including polyethylene. In Fig. 6 we see that application of the $E$-field decreases the Ps yield, but down to a certain limit, which is determined by the $\mathrm{e}_{i n}^{+}$contribution to Ps formation.

Is it known (and Fig. 6 also demonstrates it) that halogen-containing organic compounds are good acceptors of epithermal track electrons. Since the latter are Ps precursors, the addition of such scavengers reduces the Ps yield down to a certain limit (this is called partial inhibition), Fig. 6.

However, if the halogens bound track electrons only weakly, thus suppressing ion-electron recombination, the Ps yield can eventually be increased.

It was also found that halogen atoms in some cases significantly reduces the $\mathrm{e}^{+}$mobility (for example, $\mathrm{C}_{6} \mathrm{~F}_{6}$, see Fig. 3). Perhaps, they capture positrons into a shallow trap as well, which, nevertheless, does not prevent Ps formation.

\section{Conclusion}

Ps formation in molecular media is a complex process that depends on many parameters. This is why there is 
no complete theory of this process. Some models pick up only some its features. Nevertheless, comparison of these models with experiment allows to make some conclusions: (1) the formation of Ps takes place on a terminal part of the $\mathrm{e}^{+}$track ( $\mathrm{e}^{+}$blob), containing not one or two, but several tens of ion-electron pairs; (2) the Onsager theory is not suitable for describing Ps formation, since it leads to physically meaningless values of the parameters involved. Instead, we should use the diffusion-recombination model $\left(\mathrm{e}^{+}\right.$blob model), which is based on equations of inhomogeneous chemical kinetics; (3) the large difference in mobilities of positively charged ions and track electrons leads to the appearance of a local electric field within the $\mathrm{e}^{+}$blob. It prevents escape of electrons from ions, but facilitates escape of the positron out of the blob. As a result, by the end of thermalization the positrons can be divided into two fractions: "in-blob" positrons and "out-of-blob" positrons. The positrons which thermalized inside blobs are mostly involved in Ps formation, and it is difficult to act on this process by an external electric field. On the other hand, the external field easily prevents the return of $\mathrm{e}_{\text {out }}^{+}$back to the blob and thereby decreases the Ps formation probability.

\section{References}

[1] Radiation Chemistry: From Basics to Applications in Material and Life Sciences, Eds. M. SpotheimMaurizot, M. Mostafavi, Th. Douki, J. Belloni, EDP Sciences, 2008.
[2] O. Mogensen, Positron Annihilation in Chemistry, Springer-Verlag, Berlin 1995.

[3] S.V. Stepanov, V.M. Byakov, D.S. Zvezhinskiy, G. Duplâtre, R.R. Nurmukhametov, P.S. Stepanov, Adv. Phys. Chem. 2012, 431962 (2012).

[4] Z. Francis, S. Incerti, M. Karamitros. H.N. Tran, C. Villagrasa, NIM Phys. Res. B 269, 2307 (2011).

[5] S.V. Stepanov, V.M. Byakov, D.S. Zvezhinskiy, G. Duplâtre, L.Yu. Dubov, P.S. Stepanov, Yu.D. Perfiliev, L.A. Kulikov, J. Phys. Conf. Series 674, 012018 (2016).

[6] V.M. Byakov, S.V. Stepanov, J. Radioanal. Nucl. Chem. 210, 371 (1996).

[7] C.L. Wang, Y. Kobayashi, K. Hirata, Radiat. Phys. Chem. 58, 451 (2000).

[8] T. Hirade, F.H.J. Maurer, M. Eldrup, Radiat. Phys. Chem. 58, 465 (2000).

[9] S.V. Stepanov, V.M. Byakov, Y. Kobayashi, Phys. Rev. B 72, 054205 (2005).

[10] S.V. Stepanov, C.-L. Wang, Y. Kobayashi, V.M. Byakov, K. Hirata, Radiat. Phys. Chem. 58, 403 (2000).

[11] S.V. Stepanov, V.M. Byakov, J. Chem. Phys. 116 6178 (2002).

[12] A. Hummel, W.F. Schmidt, Radiat. Res. Rev. 5, 199 (1974).

[13] S. Linderoth, I.K. Mackenzie, S. Tanigawa, Phys. Lett. A 107, 409 (1985).

[14] Y. Ito, Radiat. Phys. Chem. 68 , 403 (2003).

[15] V. Storchak, J.H. Brewer, G.D. Morris, Phys. Rev. Lett. 75, 2384, (1995). 